\title{
Photomechanical Energy Conversion of Photoresponsive Fibers Exhibiting Bending Behavior
}

\author{
Kazuya Nakata, ${ }^{1,2}$ Yosuke Ishikawa, ${ }^{1,3}$ Munetoshi Sakai, ${ }^{1}$ Baoshun Liu, ${ }^{1}$ Tsuyoshi Ochiai, ${ }^{1,2}$ \\ Hideki Sakai, ${ }^{2,3}$ Taketoshi Murakami, ${ }^{1}$ Masahiko Abe, ${ }^{2,3}$ and Akira Fujishima ${ }^{1,2}$ \\ ${ }^{1}$ Photocatalyst Group, Kanagawa Academy of Science and Technology, KSP Building East 412, 3-2-1 Sakado, Takatsu-ku, Kanagawa, \\ Kawasaki 213-0012, Japan \\ ${ }^{2}$ Division of Energy and Environment Photocatalyst, Research Institute for Science and Technology, Tokyo University of Science, \\ 1-3 Kagurazaka, Tokyo, Shinjuku-ku 162-8601, Japan \\ ${ }^{3}$ Department of Pure and Applied Chemistry, Faculty of Science and Technology, Tokyo University of Science, Noda 278-8510, Japan
}

Correspondence should be addressed to Kazuya Nakata, pg-nakata@newkast.or.jp

Received 12 June 2012; Revised 13 August 2012; Accepted 14 August 2012

Academic Editor: Vincenzo Augugliaro

Copyright () 2012 Kazuya Nakata et al. This is an open access article distributed under the Creative Commons Attribution License, which permits unrestricted use, distribution, and reproduction in any medium, provided the original work is properly cited.

\begin{abstract}
Photoresponsive fibers based on poly(acrylamide) (PAA) with methylene blue (MB) dye were prepared. All semicircular fibers show bending towards the direction of the flat surface of the fiber when illuminated. The fibers recover their initial shape when the illumination stops. The fiber is heated upon illumination and cooled to room temperature once the illumination is stopped. The fiber also is sensitive to humidity, showing bending behavior towards the direction of the flat surface of the fiber upon changing the humidity. The mechanical energy of the PAA/MB fiber is approximately $0.6 \mathrm{mN}$ for the bending direction when it is illuminated. A possible mechanism for the bending behavior is as follow: (1) the fiber is heated upon illumination because of the photothermal effect, (2) the fiber loses water molecules, (3) the fiber shrinks; bending towards the direction of the flat surface of the fiber occurs because of a difference in the shrinkage for the flat surface and the other side of the fiber. Finally, we demonstrated that a PP ball $(1.5 \mathrm{mg})$ can be moved by the mechanical energy produced by the changing shape of the fiber upon illumination.
\end{abstract}

\section{Introduction}

Utilization of solar energy is a key factor for development of a sustainable society. Among a number of approaches for solar energy conversion, photomechanical energy conversion has significant advantages such as direct energy conversion from photo to mechanical, which may potentially lead to high energy conversion efficiency, no cable for working, and space-saving. Typical photomechanical materials that change shape in response to light are liquid crystalline elastomer films based on azobenzene derivatives [1-14]. These materials show bending behavior upon illumination because the structure of azobenzene derivative molecules in the film changes from trans to cis form, which generates mechanical energy. Although these materials have attracted much attention because they can be applied as photodriven motors [9], actuators $[1,3,4,6,8,10,11,15-17]$, and new types of solar energy conversion systems $[2,5,7,11-14,16,18$,
19], they need multiple synthetic procedures followed by rubbing to align the azobenzene molecules, which makes them impractical for many applications.

Materials exhibiting a volume change in response to light are of interest [20-22]. For example, hydrogels that show a volume change upon the application of light have been reported previously [21-23]. The basic mechanism for the volume change is a solvent absorption/desorption process. Suzuki and Tanaka reported that a polymer gel fiber consisting of polymer formed by copolymerization of $\mathrm{N}$-isopropylacrylamide and $\mathrm{N}, \mathrm{N}^{\prime}$-methylenebisacrylamide with copper chlorophilline as a dye shows reversible volume changes upon illumination. The diameter of the fiber varies with the intensity of light [22]. Tatsuma et al. reported that hydrogels based on hygroscopic polymers such as poly(acrylamide) (PAA) and poly(acrylic acid) with metal ions, $\mathrm{Cu}^{+}$or $\mathrm{Ag}^{+}$ and $\mathrm{TiO}_{2}$, show reversible volume changes upon illumination with visible or UV light $[20,21]$. Although preparing 


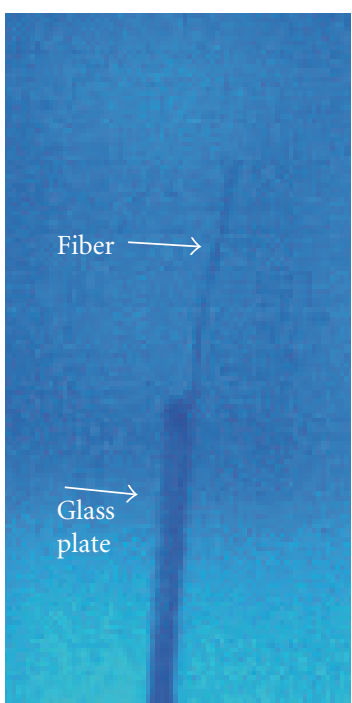

(a)

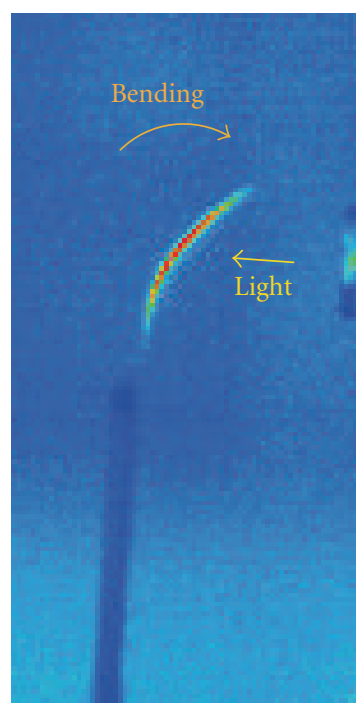

(b)

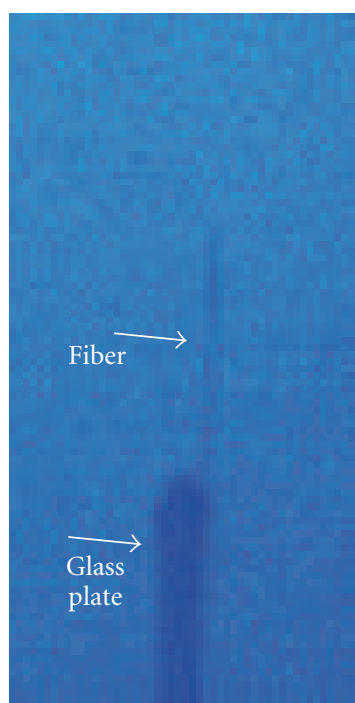

(c)

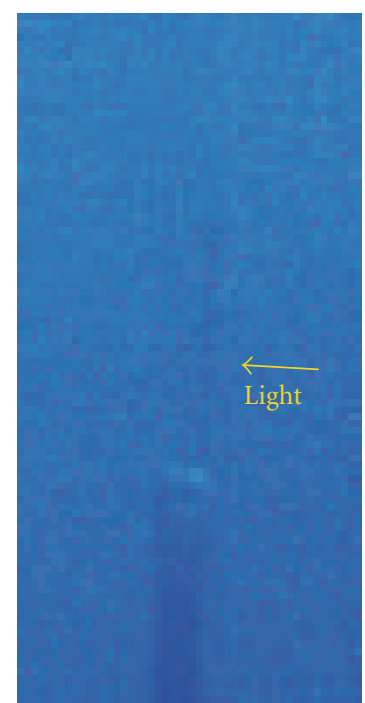

(d)

Figure 1: Thermographic images of the PAA/MB fiber (a) before and (b) after illumination, and the PAA fiber (c) before and (d) after illumination.

these materials is simple, there is a drawback for their practical application, because the absorption/desorption process can only be achieved in a solvent and not in air. Another drawback is that the volume change is slow. Thus, materials showing a rapid volume change in air are desired.

In this work, we prepared photoresponsive fibers based on polymers with $\mathrm{MB}$ as a dye. Rapid reversible bending behavior of the fibers upon illumination and also upon humidity changes was observed. Mechanical energies for the semicircular-shaped and round-shaped PAA/MB fibers or round-shaped polystyrene (PS)/MB fibers upon illumination are measured. We finally propose a mechanism for the bending behavior of the fibers and demonstrate photomechanical energy conversion using a PAA/MB fiber and a PP ball.

\section{Experimental Procedure}

The fibers were prepared according to the previous report [23]. To obtain PAA/MB, a viscous water solution $(10 \mathrm{~mL})$ containing PAA (1 g, MW: 5000000-6000000, Polysciences, Inc.) was added with either MBs $(32 \mathrm{mg}, 0.1 \mathrm{mmol}$ ) followed by stirring for $1 \mathrm{~h}$. A part of the resulting viscous liquid was anchored using a stick and then pulled up to readily form fibers of 100 to $1000 \mathrm{~mm}$ in diameter. The fibers were placed on a polystyrene substrate to dry for $24 \mathrm{~h}$ at room temperature at a humidity of $50 \%$ to obtain semicircularshaped fibers. To form round-shaped PAA/MB fiber, the fiber was prepared according to the above procedures without having been placed on the polystyrene substrate. A round PS/MB fiber was prepared according to the above procedures using PS (Mw: 350000, Aldrich) and DMF (10 mL) instead of PAA and water, respectively, without having been placed on the polystyrene substrate.

\section{Results and Discussion}

Figures 1(a) and 1(b) show thermographic images of the PAA/MB fiber before and after illumination. The fiber was fixed on the glass plate and then illuminated from the right side of the figure. The temperature of the fiber was $26.4^{\circ} \mathrm{C}$ before illumination and increased to $38.5^{\circ} \mathrm{C}$ at the center of the fiber after illumination. This increase of the temperature is probably because of the photo-thermal effect. The MB dye should absorb visible light because MB has an absorbance in the visible light range [23]. Then photo energy should be transformed into thermal energy. The increase of the temperature was seen every time an MB-containing fiber was illuminated; photo-bending behavior was also seen every time. This suggests that the increase in temperature and bending behavior should be related. When the light was turned off, the temperature recovered to the initial temperature and the fiber recovered to its initial shape. When we used fibers without dye, the increase of the temperature was not observed (Figures 1(c) and 1(d)).

The fibers based on the hygroscopic polymer, PAA, clearly show changes in their shapes upon humidity changes. Figure 2 shows photographs of the fiber at $90 \%$ and $30 \%$ humidity. The shape of the fiber was bent towards the flat surface of the fiber when the humidity changed from $90 \%$ to $30 \%$. When the humidity returned to $90 \%$, the fiber recovered its initial shape. The change in shape of the fiber is repeatable. Rapid changes in shape of the fiber were produced by flowing dry air (humidity almost $0 \%$ ); the fiber then recovered to its initial position when exposed to moist air (humidity, approximately 90\%).

A differential scanning calorimetry trace for PAA/MB fiber is shown in Figure 3. An endothermic peak was 


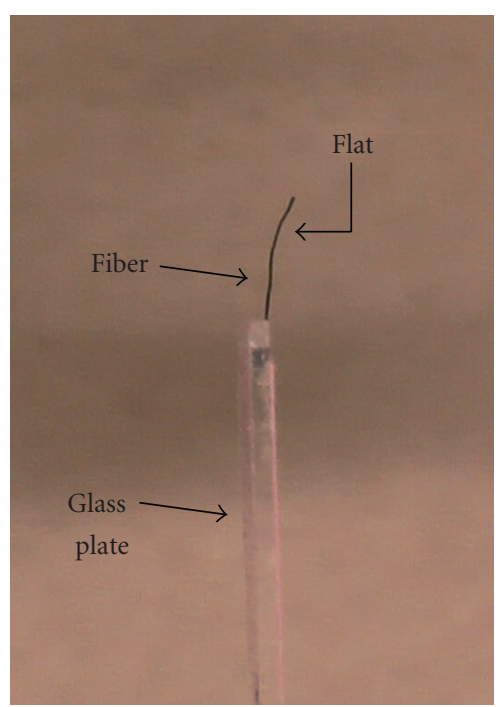

(a)

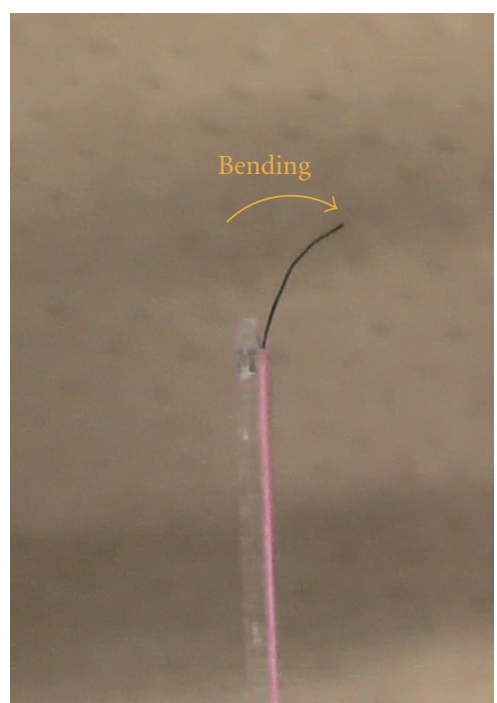

(b)

Figure 2: Photos of PAA/MB fiber at humidities of (a) $90 \%$ and (b) 30\%.

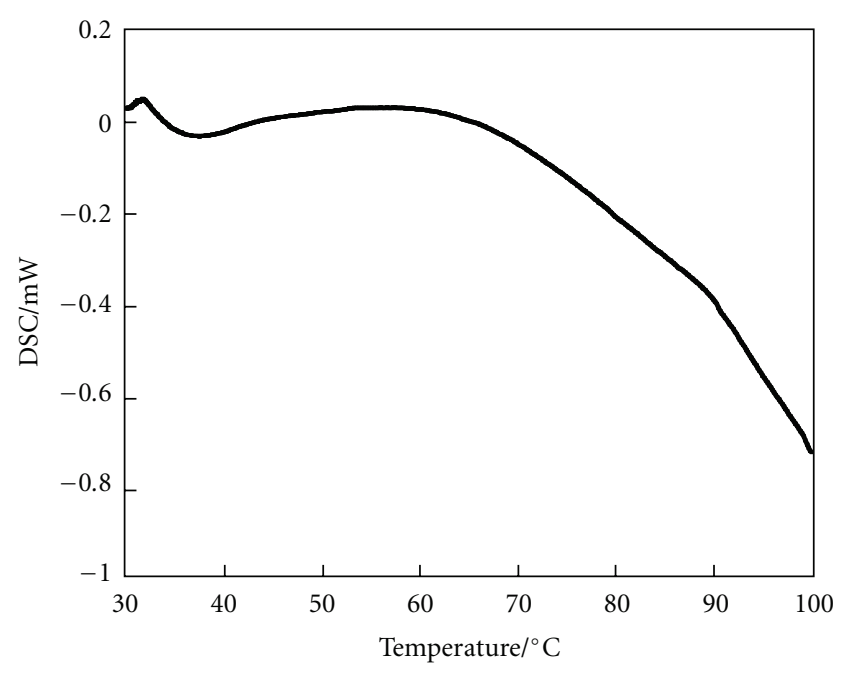

FIgURE 3: Differential scanning calorimetry of PAA/MB fiber.

observed at $37.4^{\circ} \mathrm{C}$, which can be attributed to the loss of water molecules from the PAA fibers. The temperature of the endothermic peak is near the temperature measured at the center of the sample which is bending with illumination, $38.5^{\circ} \mathrm{C}$. The loss of weight at the temperature is also seen in thermogravimetric analysis (Supplementary Figure S1 available online at doi:10.1155/2012/574124). This implies that upon illumination, water molecules are released from the fiber.

We measured the mechanical force exerted by the semicircular shaped PAA/MB fiber when it was illuminated. First, we measured the force in the direction perpendicular to the fiber (bending force) as shown in Figure 4(a). When the fiber was illuminated, approximately $0.6 \mathrm{mN}$ was generated in this direction, with a response time of approximately $0.6 \mathrm{sec}$. Then, when the illumination was turned off, the bending force disappeared within approximately $0.7 \mathrm{sec}$. Generation and disappearance of the mechanical force can be cycled by turning the illumination on and off.

We next tried to observe the mechanical force produced by a round $\mathrm{PAA} / \mathrm{MB}$ fiber in the direction parallel to the fiber upon illumination as shown in Figure 4(b). When the fiber was illuminated, the fiber showed a small expansion within $0.2 \mathrm{sec}$ and then shrank with a force of approximately $0.6 \mathrm{mN}$. For reference, a PS/MB fiber was also illuminated; this fiber expanded without the subsequent shrinkage seen in the PAA/MB fiber. These contrasting results for PAA/MB and $\mathrm{PS} / \mathrm{MB}$ are probably originated from different mechanisms for generation of the mechanical force in these two fibers. For the PAA/MB fiber, we first observed a small expansion as the fiber expanded due to thermal expansion because of a photo-thermal effect. Then the fiber shrank because of loss of water molecules. In contrast, the PS/MB fiber shows only expansion, since PS is not a hygroscopic polymer, and does not show loss of water (Figure 4(c)).

Based on the above results, we propose the following mechanism for the bending of the PAA/MB fiber as shown in Figure 5: (1) first, the fiber is heated when it is illuminated, because of the photo-thermal effect; (2) then, water molecules from the PAA/MB fiber are released to air; (3) finally, the fiber shrinks and shows bending behavior, probably as a result of different shrinkages of the flat surface and the other side of the fiber. Recovery of the shape of the fiber when the light is turned off and the fiber cools probably 


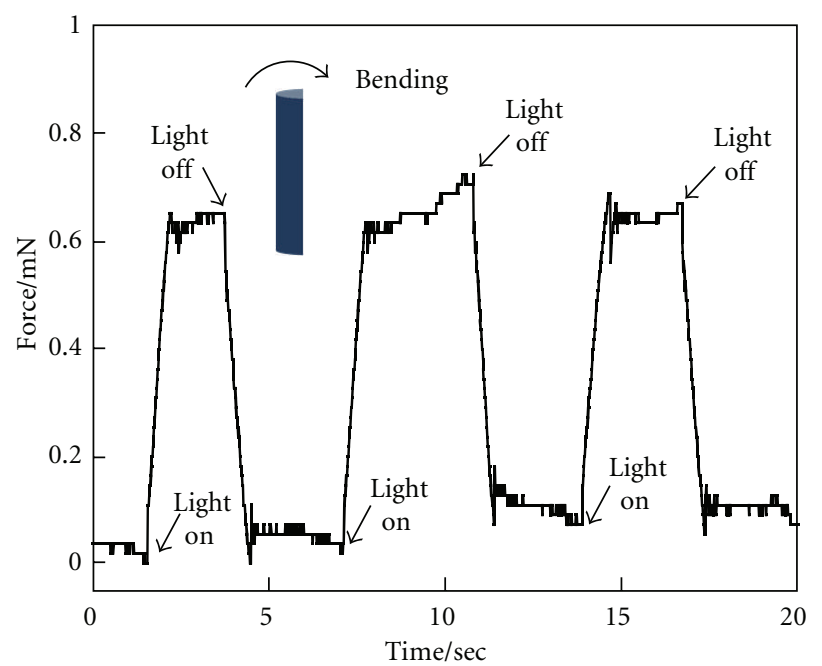

(a)

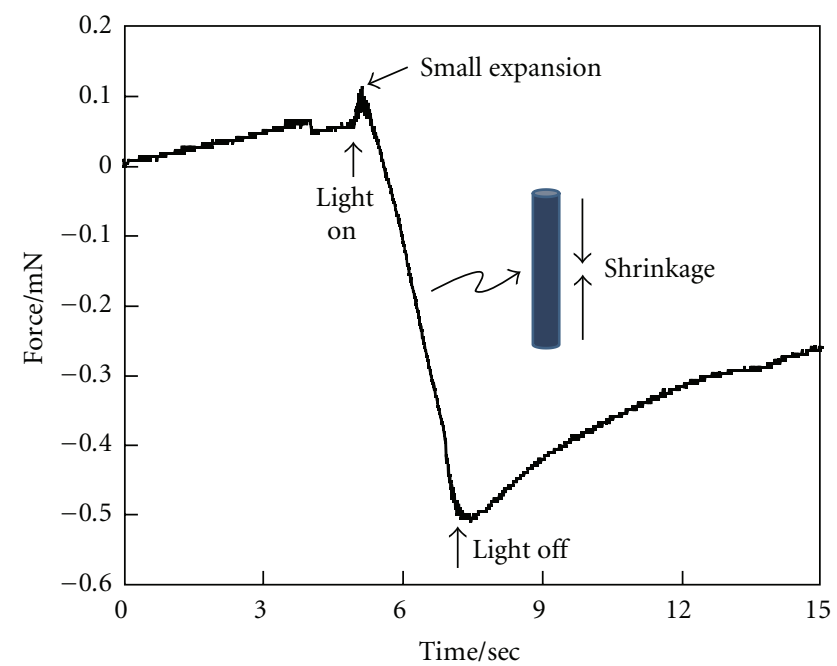

(b)

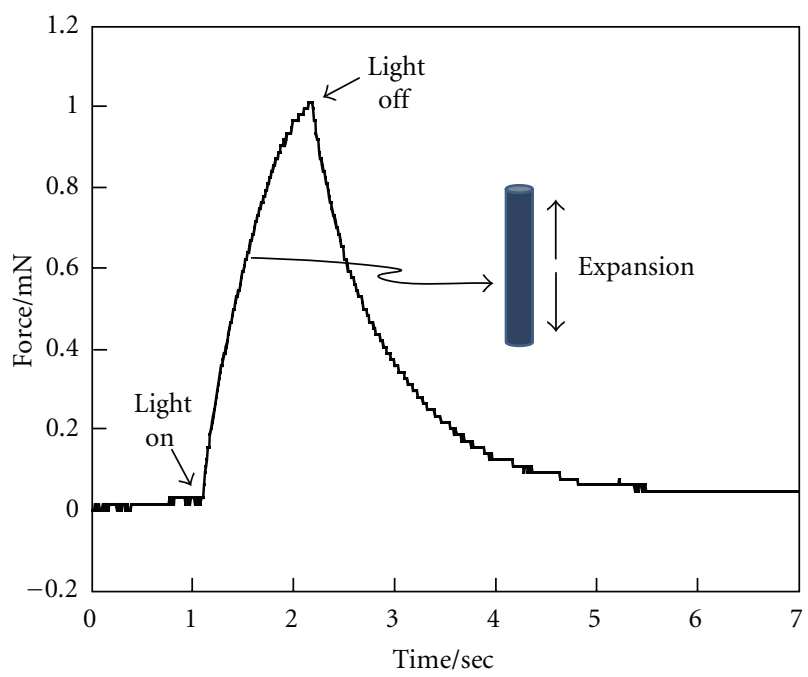

(c)

FIgURE 4: Measurements of mechanical force for (a) semicircular PAA/MB fiber, (b) round PAA/MB fiber, and (c) round PS/MB fiber.

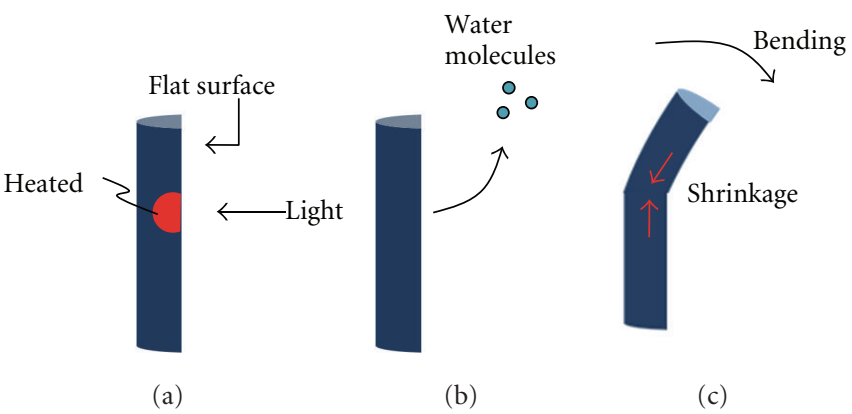

FIGURE 5: Mechanism of bending behavior of the PAA/MB fiber. 


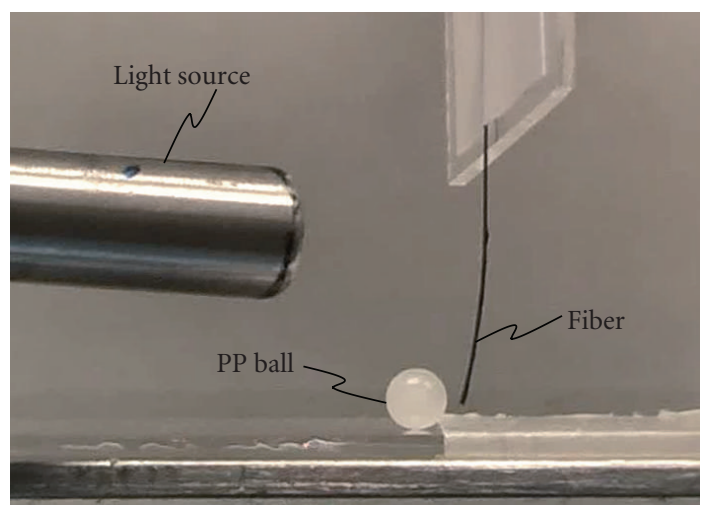

(a)

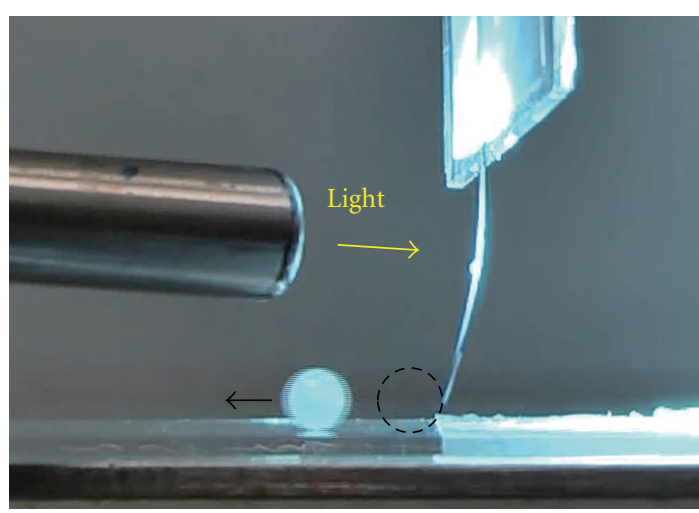

(b)

Figure 6: Photos demonstrating photo to mechanical energy conversion using a PAA/MB fiber and a PP ball (a) before and (b) after illumination.

involves absorption of water molecules from air, and elastic recovery of the PAA.

Finally, we demonstrated photomechanical energy conversion using a semi-circular PAA/MB fiber. We fixed the fiber on a glass plate, and a PP ball $(1.5 \mathrm{mg})$ was placed on the substrate as shown in Figure 6. After the fiber was illuminated, the fiber bent and pushed the ball from the right; the ball then moved, which meant that the photomechanical energy conversion was achieved.

\section{Conclusion}

In conclusion, we have prepared photoresponsive fibers based on hygroscopic polymers with $\mathrm{MB}$ as a dye. The fibers show bending behavior to the direction perpendicular to the fiber when illuminated. The fiber recovers its initial shape when the illumination is stopped. The fiber is heated upon illumination and cooled to room temperature once the illumination is stopped. The fiber is also sensitive to humidity, showing bending in response to humidity changes. The mechanical energy for the fiber is $0.6 \mathrm{mN}$ in the bending direction when it is illuminated. A possible mechanism for the bending behavior is as follows: (1) the fiber is heated upon illumination because of the photo-thermal effect, (2) the fiber loses water molecules, and (3) the fiber exhibits shrinkage and shows bending behavior because of a difference in shrinkage between the flat surface and the other surface of the fiber. Finally, we demonstrated that a PP ball (15 mg) can be moved by the mechanical energy produced by the shape change of the fiber upon illumination.

\section{Acknowledgment}

This research was supported by a Grant-in-Aid for Scientific Research in the priority area "New Frontiers in Photochromism (no. 471)" from the Ministry of Education, Culture, Sports, Science, and Technology (MEXT), Japan.

\section{References}

[1] T. Ikeda, J. I. Mamiya, and Y. Yu, "Photomechanics of liquidcrystalline elastomers and other polymers," Angewandte Chemie, vol. 46, no. 4, pp. 506-528, 2007.

[2] T. Ikeda, M. Nakano, Y. Yu, O. Tsutsumi, and A. Kanazawa, "Anisotropic bending and unbending behavior of azobenzene liquid-crystalline gels by light exposure," Advanced Materials, vol. 15, no. 3, pp. 201-204, 2003.

[3] C. J. Barrett, J. I. Mamiya, K. G. Yager, and T. Ikeda, "Photomechanical effects in azobenzene-containing soft materials," Soft Matter, vol. 3, no. 10, pp. 1249-1261, 2007.

[4] H. J. Choi, K. U. Jeong, L. C. Chien, and M. H. Lee, "Photochromic 3-dimensional actuator based on an uncrosslinked liquid crystal elastomer," Journal of Materials Chemistry, vol. 19, no. 38, pp. 7124-7129, 2009.

[5] C. D. Eisenbach, "Isomerization of aromatic azo chromophores in poly(ethyl acrylate) networks and photomechanical effect," Polymer, vol. 21, no. 10, pp. 1175-1179, 1980.

[6] K. D. Harris, R. Cuypers, P. Scheibe et al., "Large amplitude light-induced motion in high elastic modulus polymer actuators," Journal of Materials Chemistry, vol. 15, no. 47, pp. 50435048, 2005.

[7] M. Kondo, Y. Yu, and T. Ikeda, "How does the initial alignment of mesogens affect the photoinduced bending behavior of liquid-crystalline elastomers?" Angewandte Chemie, vol. 45, no. 9, pp. 1378-1382, 2006.

[8] C. L. Van Oosten, C. W. M. Bastiaansen, and D. J. Broer, "Printed artificial cilia from liquid-crystal network actuators modularly driven by light," Nature Materials, vol. 8, no. 8, pp. 677-682, 2009.

[9] M. Yamada, M. Kondo, J. I. Mamiya et al., "Photomobile polymer materials: towards light-driven plastic motors," Angewandte Chemie, vol. 47, no. 27, pp. 4986-4988, 2008.

[10] T. Yoshino, M. Kondo, J.-I. Mamiya, M. Kinoshita, Y. Yu, and T. Ikeda, "Photomobile polymer materials - towards lightdriven plastic motors," Advanced Materials, vol. 12, pp. 13611363, 2010.

[11] Y. Yu and T. Ikeda, "Soft actuators based on liquid-crystalline elastomers," Angewandte Chemie, vol. 45, no. 33, pp. 54165418, 2006. 
[12] Y. Yu, T. Maeda, J. I. Mamiya, and T. Ikeda, "Photomechanical effects of ferroelectric liquid-crystalline elastomers containing azobenzene chromophores," Angewandte Chemie, vol. 119, pp. 899-901, 2007.

[13] Y. Yu, M. Nakano, and T. Ikeda, "Directed bending of a polymer film by light," Nature, vol. 425, no. 6954, p. 145, 2003.

[14] Y. Yu, M. Nakano, A. Shishido, T. Shiono, and T. Ikeda, "Effect of cross-linking density on photoinduced bending behavior of oriented liquid-crystalline network films containing azobenzene," Chemistry of Materials, vol. 16, no. 9, pp. 1637-1643, 2004.

[15] S. V. Ahir and E. M. Terentjev, "Photomechanical actuation in polymer-nanotube composites," Nature Materials, vol. 4, no. 6, pp. 491-495, 2005.

[16] S. Kobatake, S. Takami, H. Muto, T. Ishikawa, and M. Irie, "Rapid and reversible shape changes of molecular crystals on photoirradiation," Nature, vol. 446, no. 7137, pp. 778-781, 2007.

[17] H. Koshima, N. Ojima, and H. Uchimoto, "Mechanical motion of azobenzene crystals upon photoirradiation," Journal of the American Chemical Society, vol. 131, no. 20, pp. 6890-6891, 2009.

[18] O. M. Tanchak and C. J. Barrett, "Light-induced reversible volume changes in thin films of azo polymers: the photomechanical effect," Macromolecules, vol. 38, no. 25, pp. 1056610570, 2005.

[19] G. V. D. Veen and W. Prins, "Where doctors draw the line," Nature, vol. 230, pp. 70-72, 1971.

[20] K. Takada, T. Miyazaki, N. Tanaka, and T. Tatsuma, "Threedimensional motion and transformation of a photoelectrochemical actuator," Chemical Communications, no. 19, pp. 2024-2026, 2006.

[21] T. Tatsuma, K. Takada, and T. Miyazaki, "UV-light-induced swelling and visible-light-induced shrinking of a $\mathrm{TiO}_{2}$-containing redox gel," Advanced Materials, vol. 19, no. 9, pp. 12491251, 2007.

[22] A. Suzuki and T. Tanaka, "Phase transition in polymer gels induced by visible light," Nature, vol. 346, no. 6282, pp. 345-346, 1990.

[23] K. Nakata, Y. Ishikawa, M. Sakai et al., "Anisotropic photomechanical motion of semicircular-shaped microfibers that contain dyes," Chemistry Letters, vol. 40, pp. 1229-1230, 2011. 


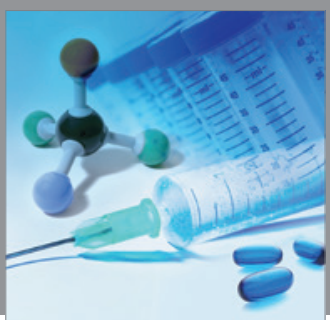

International Journal of

Medicinal Chemistry

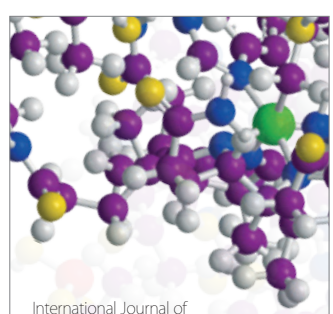

Carbohydrate Chemistry

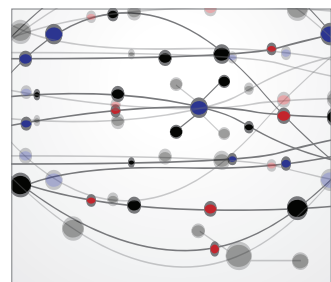

The Scientific World Journal
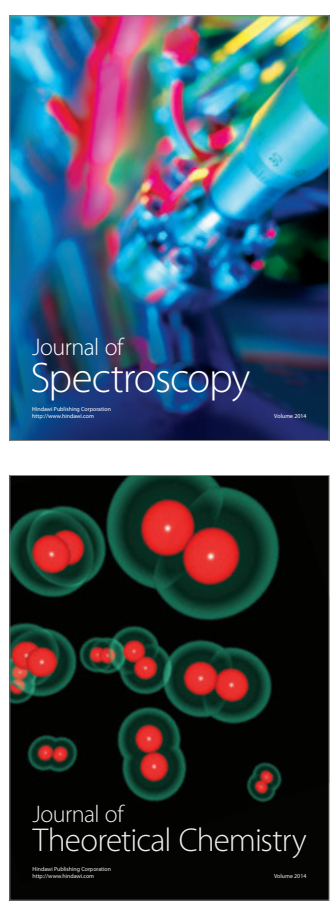
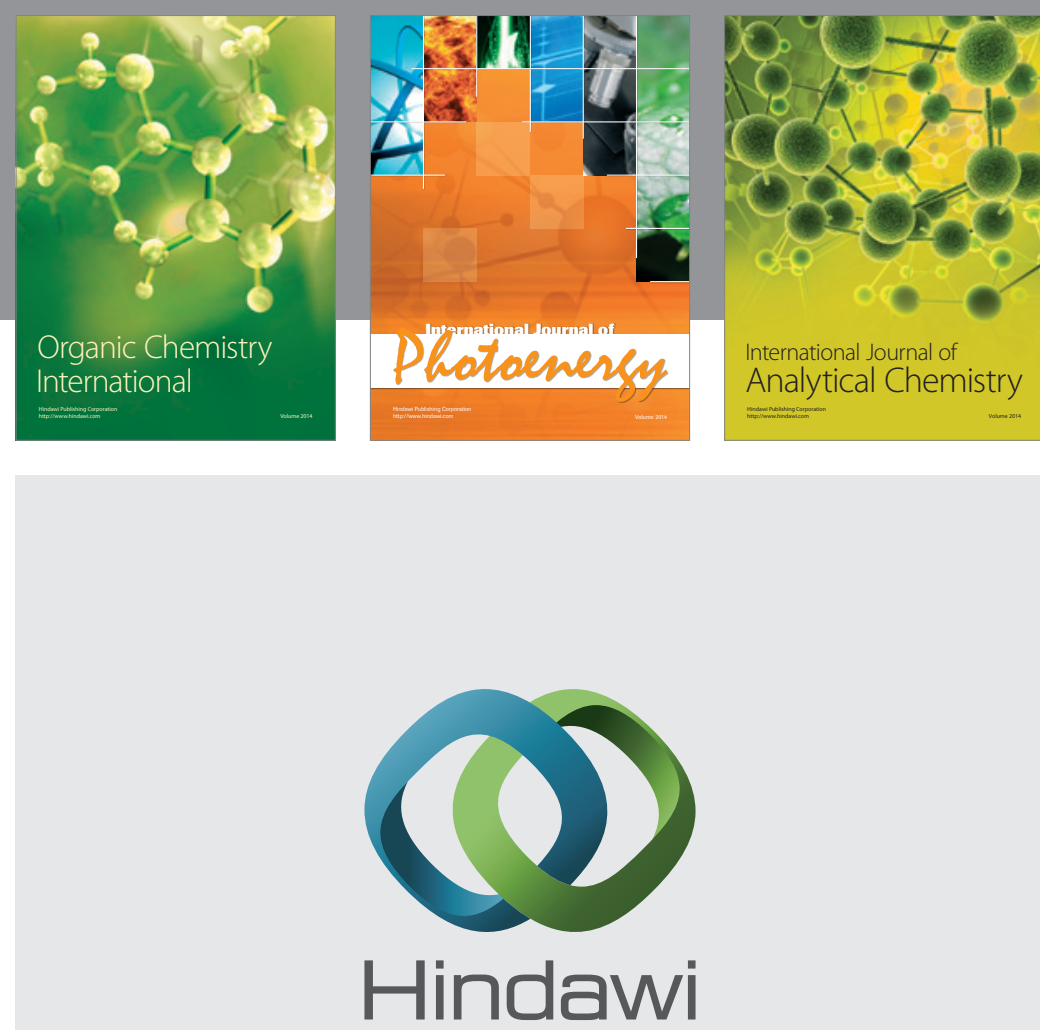

Submit your manuscripts at

http://www.hindawi.com
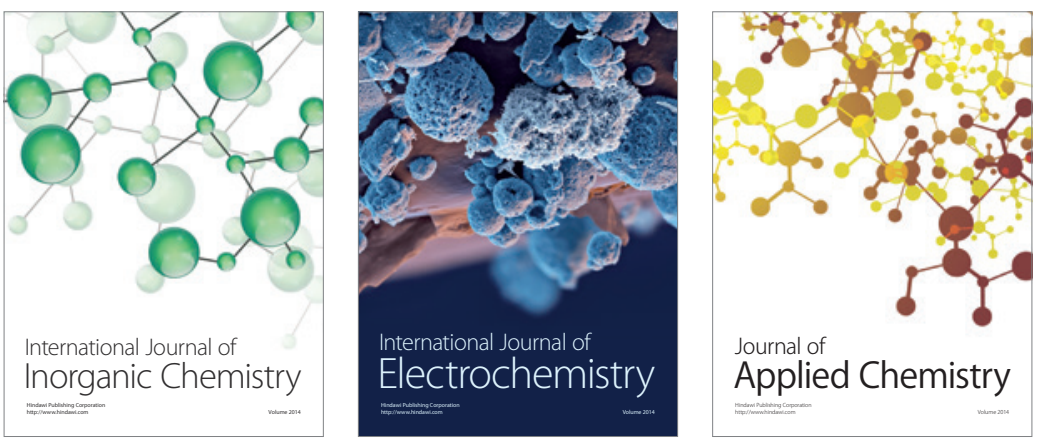

Journal of

Applied Chemistry
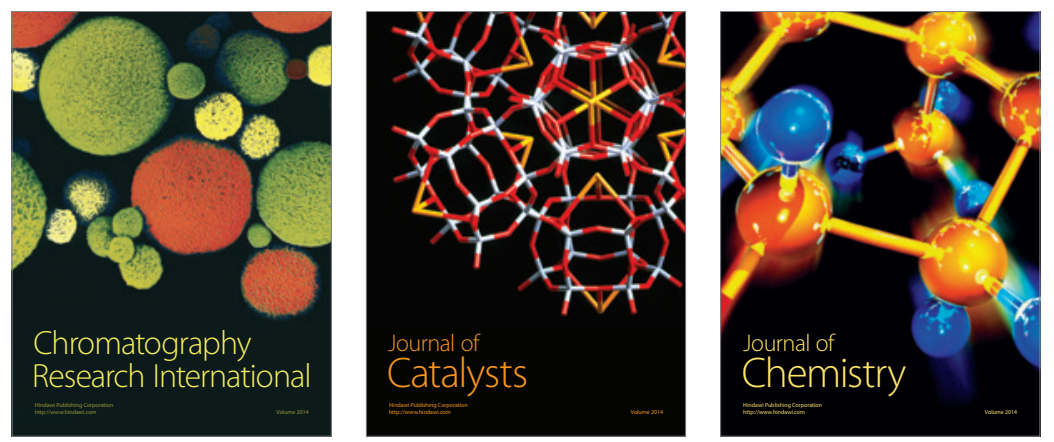
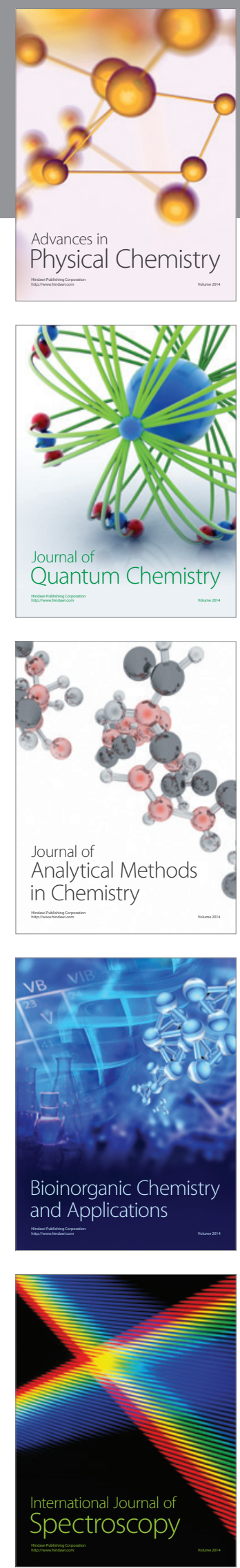